\title{
Comparison of nanosilver inhibitory effects growth between Aspergillus niger and E. coli
}

\author{
N. Naghsh ${ }^{*}$, M. Safari and P. Hajmehrabi \\ Department of Biology, Falavarjan branch, Islamic Azad University, Falavarjan, Iran \\ *n_naghsh@yahoo.com, naghsh@iaufala.ac.ir
}

\begin{abstract}
Nanotechnology is employed in new and specific method for disease therapy. The shape and size of nano-particles are also important to serve the purposes. In this investigation, a comparison of inhibitory effects on Aspergillus nigerand $E$. coli by nanosilver in 100, 200,300, 400, and 500 ppm concentration was carried out. For this purpose, inseminated blank antibiogram disks were placed on bacterial culture over a nutrient agar environment of 0.5 Mac Farland`s standard. After an interval of first, second, and eighth days, the inhibitory zone was measured. In case of Aspergillus niger, PDA medium was used. The fungal growth was more sensitive to nanosilver than E.coli. Hence, nanosilver particles with 50, 100, and 150-ppm concentration were added to the culture medium for A.niger. After 6, 7, and 8 days, changes of growth, diameter and number of colonies were measured in treatment and control groups. In this investigation, nanosilver with 400-ppm concentration was more effective than the other concentrations for inhibiting E.coli. Effective time for induction of inhibitory effects on E.coli was 3-day post treatment with silver nano-particles. Effective dose of nanosilver concentration for growth inhibition of Aspergillus niger was 150ppm 8 day post treatment with silver nano-particles. Investigation of inhibitory effects for nanosilver concentration in Aspergilus niger and $E$. coli are examples of functional effects of new nano-biotechnlogy. These could be suggested in the therapy for human bacterial and fungal diseases.
\end{abstract}

Keywords: Nanosilver, nanobiotechnology, Aspergilus niger, E. coli.

Introduction

Nanoparticles are small particles that have many application in medical and biology development. There is lack of information concerning the human health and environmental implications of manufactured nanometals (Bhainsa \& DSouza, 2006). The nanosize results in specific physicochemical properties that may different from those of the bulk substance or particles of larger size. This effect is mainly attributed to high surface area to volume ratio, which potentially results in high reaction activity. Silver is one of these materials that have antibacterial and antifungal effects. Nanosilver is an effective killing agent against a broad spectrum of Gram negative and Gram-positive bacteria and antibioticresistant strains. In this investigation, $E$. coli is one of negative gram bacteria that are studied as a model of these bacteria.

This paper highlights the comparison of nanosilver inhibitory effects on Aspergilus niger. Since these are relatively new particles. The shape and size of nanoparticles are also considered important. For this reason, in this investigation, $A$. niger were treated with spherical shape and 4 nanometer diameter nanosilver particles. In many studies, nanosilver antibacterial effects have been reviewed. Exact reports about antibacterial effects and antifungal effects of these nanoparticles have not been seen.

Investigators could suggest that the resultant structural change in the cell membrane could cause an increase in cell permeability, leading to an uncontrolled transport through the cell membrane and ultimately apoptosis. It has also been proposed, that the antibacterial mechanism of silver nanoparticles is related to the formation of free radicals and subsequent free radical-induced membrane and DNA damage. Toxic effect of the silver nanoparticles and the silver ions relate to concentration of free radicals and rate of oxidative stress reactions. Silver ions move into the cells and lead to the production of reactive oxygen species. Here, we study various aspects of nanoparticle on the growth effect on fungus $A$. niger and bacterium $E$. coli (Moudgil \& Roberts, 2006).

Beacause these organisms also cause illness on human beings, investigations on their growth inhibition would help in curbing infectious disease (Arikan et al., 2001). Thus investigations on specialized effects of silver nano particles for inhibiting fungus and bacterium physiologically would of new application for nano technology within the field of biotechnology (Arikan \& Rex, 2000).

Materials and methods

Culture of bacterial strains

In order to produce the main samples of bacteria, a vial of $E$. coli bacterium was bought from Pasture Institute of Tehran. The bactericidal experiments were carried out with gram-negative bacteria $E$. coli in NA (Nutrient Agar) medium. The nanoparticles were subsequently stored in airtight containers and serial diluted with distilled and deionizer water. In this study, proper concentrations of nanosilver for E.coli were 100, 200, 300, 400, and 500 $\mathrm{ppm}$. To the culture plate containing E.coli grown for $24 \mathrm{~h}$ on Nutrient Agar, the McFarland standard equivalent to 0.5 was inseminated to each plate (Nakagawa et al., 1999). 
Fig. 1. Zone of inhibition diameter against Escherichia coli bacteria in different times of treatment in 400 ppm concentration of nanosilver

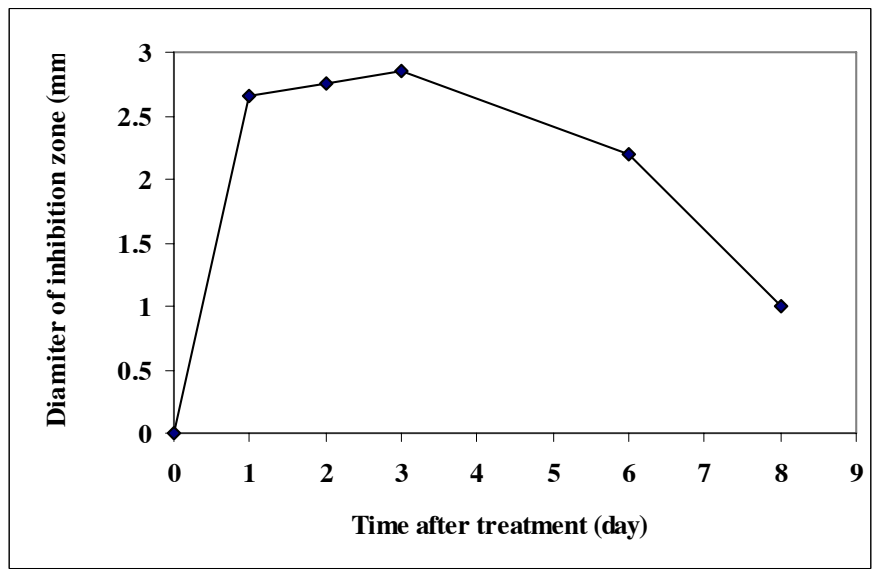

Fig. 2. Zone of inhibition diameter against Escherichia coli bacteria in different times of treatment in 500 ppm concentration of nanosilve

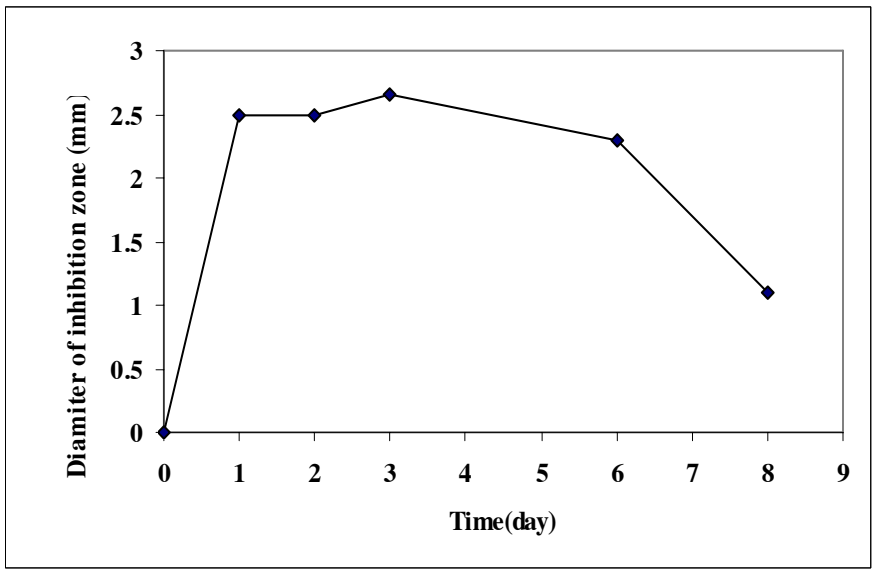

Fig. 3. Zone of inhibition diameter against Escherichia coli bacteria in different concentration of nanosilver 3-day post treatment

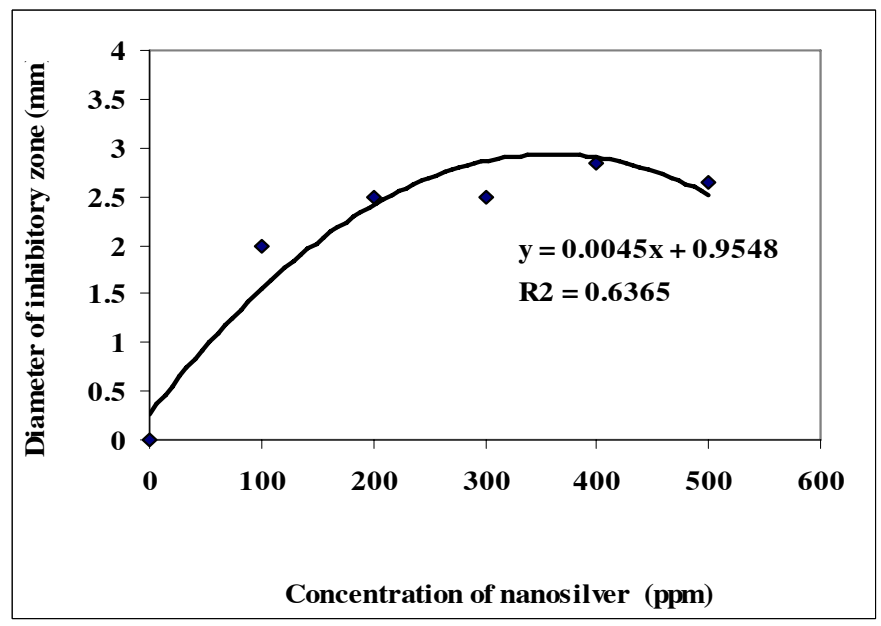

\section{Disk diffusion test}

Bacterial sensitivity to antibiotics is commonly tested using a disk diffusion test, employing antibiotic impregnated disks .A similar test with blank antibiogram disks was used in this study. A 10 microliter suspension of nanoparticles (with specific concentration) were added to blank disks and subsequently $r$ were dried in an oven for $1 \mathrm{~h}$. Diameter average of were $6 \mathrm{~mm}$. These treated disks were added to NA that enrichment to E. coli (105 CFU ml ${ }^{-1}$ ) was applied uniformly on the surface of a nutrient agar plate before placing the disks on the plate (4 per plate). The plates were incubated at $35{ }^{\circ} \mathrm{C}$ for $24 \mathrm{~h}$, after which the average diameter of the inhibition zone surrounding the disk was measured with a specific ruler with up to $1 \mathrm{~mm}$ resolution. In next step, inhibitory zones were measured during 24 and 48 hours post treatment. The mean and standard deviation (SD) reported for each type of nanoparticle and with each microbial strain was based on 4 replicates.

\section{Culture of fungal strains}

In order to produce the main samples of fungus, a vial of Aspergillus niger fungus was bought from pastor institute of Tehran. Aspergyllus niger was cultivated in PDA (Potatoes Dextrose Agar) medium. In this study, silver nanoparticles with average diameter 4.5 nanometer were bought of pars Tehran Nano nasb Installation Company. Nano silver with 50, 100, and 150-ppm concentration were added to cultivation environment. In order to make control group, redistilled water was added only to one of plates. After passing 6, 7 and 8 days, morphologic variation, diameter and the number of colonies in treated groups were compared with control groups. The number of samples repeat was considered 24 times. T-test was used to compare averages. To measure data statistically was used software SPSS program and diagrams were drawn by excel software.

Results and discussions

Maximum of inhibitory zone diameter against Escherichia coli bacteria was founded in 400ppm in different concentration of nanosilver 3-day post treatment (Fig1,2 and 3 ). Zone of inhibition (diameter) against Escherichia coli bacteria in different times of treatment in 400ppm and 500 ppm concentration of nanosilver was not significantly different (Figures 1 and 2).

Our investigations showed that the growth of Aspergillus niger in $50 \mathrm{ppm}$ concentration, was performed 6 day after treatment and continued to 8 day. Results of these investigations were shown that 6 day after treatment is minimum effective time on growth inhibition in Aspergilus niger (Figure 4). Inhibition of growth has decreased after 6 day in 50ppm. 50ppm nanosilver concentration inhibited $A$. niger (Figure 5).

In addition, there is any colony of Aspergilus niger were in culture medium in 150ppm nanosilver concentration. The diameter of colony decreased in treated groups $(6.12 \pm 0.39 \mathrm{~mm})$ compared to control groups $(58 \pm 1.39 \mathrm{~mm}) 50 \mathrm{ppm}$ nanosilver concentration 8 day post treatment that statistically has been significantly $(p=0.00)$ (Figure 6). 
Fig. 4. Nanosilver inhibitory effects on colonies diameter average of Aspergyllus niger in different concentration of nanosilver 8 day post treatment

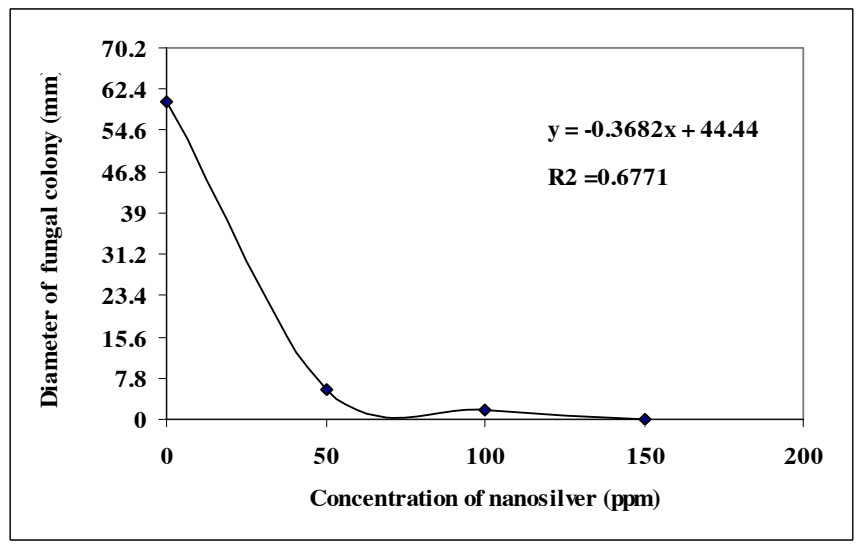

Fig. 5. Investigation of nanosilver inhibitory effects on colonies number average of Aspergyllus niger in 50 ppm nanosilver concentration in different times post treatment

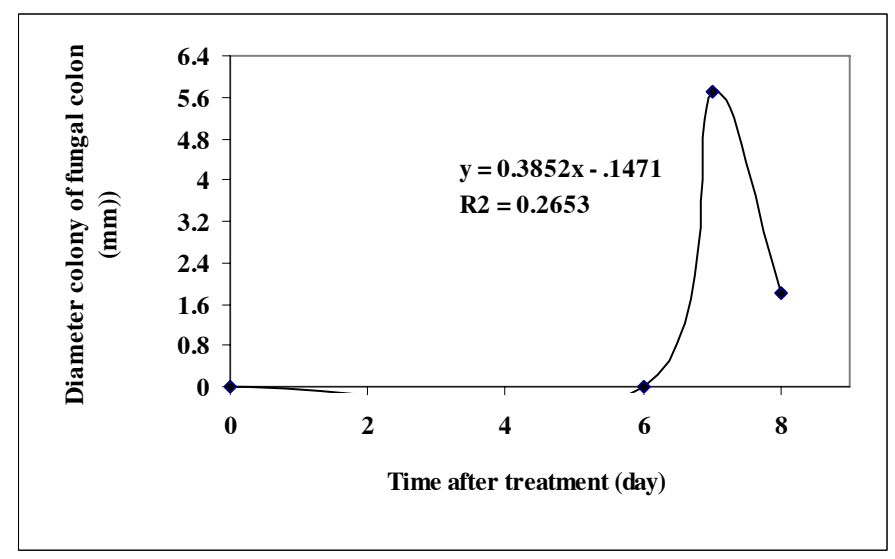

Fig. 6. Comparison of nanosilver inhibitory effects on colonies diameter average of Aspergyllus niger between control (1) and treatment (2) groups in 50 ppm nanosilver concentration 8 day post treatment

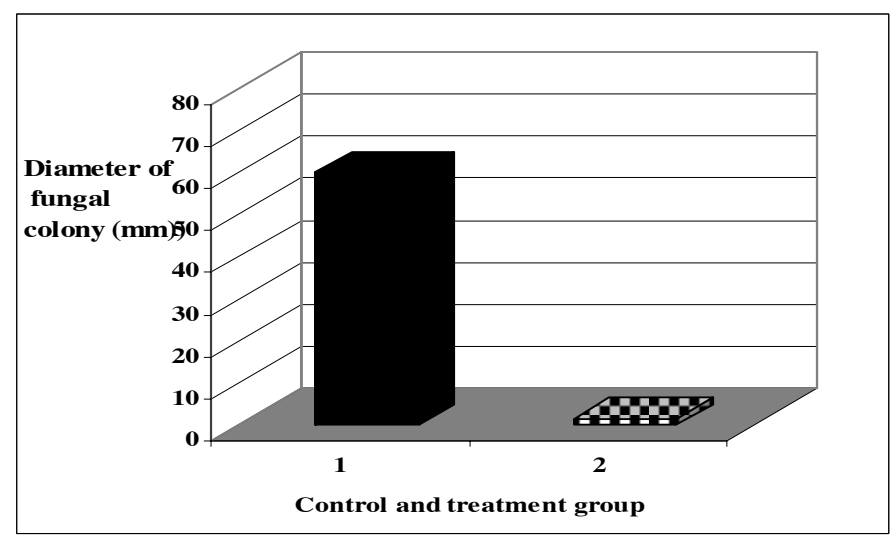

Conclusion

In this investigation, nanosilver with $400 \mathrm{ppm}$ concentration was more effective than the other concentrations for inhibiting the growth of E.coli. Effective Research article "Proceedings of The First Nano Biotechnology Conference CoIndian Society for Education and Environment (iSee) time for induction of inhibitory effects on $E$. coli was 3-day post treatment with silver nanoparticles (Figure 3). Effective dose of nanosilver concentration for growth inhibition of Aspergillus niger was $150 \mathrm{ppm} 8$ day post treatment with silver nanoparticles.

Another study showed that the colloidal silver particles, with diameter average of $4 \mathrm{~nm}$ and spherical shape was used for investigation of antibacterial effects on E. coli. Small particles exhibited higher antimicrobial activity than bigger particles. Silver ions because of lesser size, have more interfaces, and influence the cell membrane more. This study also showed that the antibacterial activity towards $E$. coli of gel hybrids containing silver nanoparticles of different sizes, i.e., 2.67, 6.63, and $21.11 \mathrm{~nm}$, were examined and compared with silver nanoparticles $(220 \mathrm{~nm})$ without any stabilization. The results showed that the silver nanoparticles of $2.67 \mathrm{~nm}$ protected by hydro gel polymer chains present an excellent antibacterial activity compared to the larger sized silver nanoparticles in the hybrid networks (Moudgil and Roberts, 2006). On the other hand, molecular mechanism provided by silver nanoparticles can be attributed to production of free radicals.

These free radicals were induced oxidative stress and program cell death in E. coli and Aspergillus niger. Some investigations showed that $\mathrm{OH}$ ion is active structures and the strongest antimicrobial elements; these free radicals attack to interior DNA of fungus and bacterium, and cause apoptosis in cells. Other mechanism is referred to silver ions practice in colloidal solution is done by converting grafts $\mathrm{S}-\mathrm{H}$ to S-Ag. In this mechanism, metaloenzymes were destroyed. Investigation of inhibitory effects for nanosilver concentration in Aspergilus niger and E. coli are microbial biologic models for investigation of inhibitory effects of nanosilver in new nanobiotechnlogy (Somayyeh et al., 2011). It is suggested that therapy of human bacterial and fungal diseases in medicine could be performed with different size, shape, concentration, and combination of nanoparticles.

References

1. Arikan S, Lozano-Chiu M, Paetznick $\mathrm{V}$ and Rex JH (2001) In vitro susceptibility testing methods for caspofungin against Aspergillus and Fusarium isolates. Antimicrob Agents Chemother. 45, 327-330.

2. Arikan S and Rex JH (2000) New agents for treatment of systemic fungal infections. Emerg. Drugs, 5, 135-160.

3. Bhainsa KC and DSouza SF (2006) Biosynthesis of silver nanoparticles using the fungus Aspergillus fumigates. Biointerfaces, 47, 160-164.

4. Moudgil BM and Roberts SM (2006) Designing a strategies for safety evaluation of nanomaterials. Part nano-interface in a microfluidic chip to probe living VI. Characterization of nanoscale particles for cells: challenges and perspectives. Toxicol. Sci.103, 64196424.

5. Nakagawa $\mathrm{Y}$, Shimazu $\mathrm{K}$, Ebihara $\mathrm{M}$ and Nakagawa $\mathrm{K}$ (1999) Aspergillus niger pneumonia with fatal pulmonary oxalosis. J. Infect Chemother. 5, 97-100.

6. Somayyeh M, Delavar HA and Motallebi A (2011) Toxicity Study of Nanosilver on Osteoblast Cancer Cell Line. Int. Nano Lett. 1,11-16.

Cindian Society for Education and Environment (iSee) 